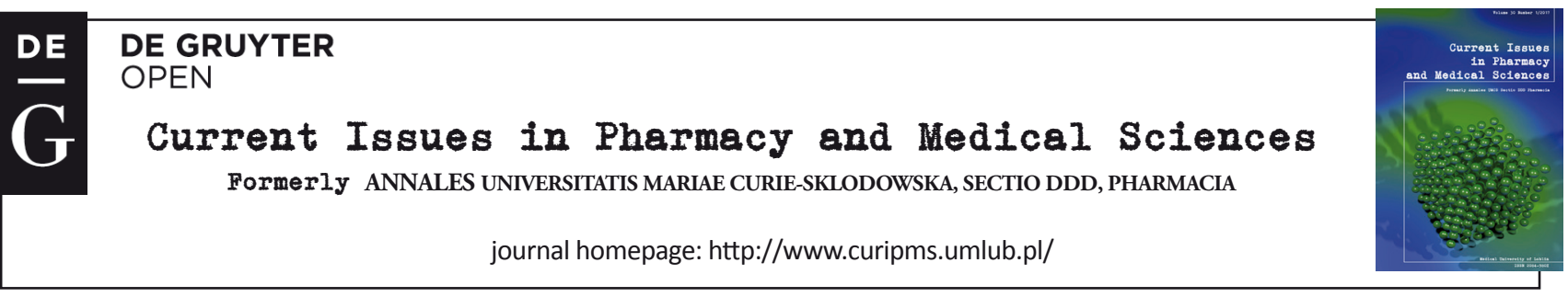

\title{
Prospective of ischemic stroke biomarkers
}

\author{
Krzysztof SzewCZak ${ }^{1 \star}$, Magdalena Niespialowska ${ }^{1}$, \\ Beata Jakubowska-Solarska ${ }^{1}$, Magdalena Chalabis ${ }^{2}$, Jacek Gawlowicz ${ }^{3}$
}

\begin{abstract}
${ }^{1}$ Department of Hematological Diagnostics, Medical University of Lublin, Antoniego Gebali 6, 20-093 Lublin, Poland
${ }^{2}$ Department of Laboratory Diagnostics, Medical University of Lublin, Chodzki 1, 20-093 Lublin, Poland

${ }^{3}$ Department of Neurology, Cardinal Rev. Wyszynski’s Voivodship Specialist Hospital in Lublin, Poland
\end{abstract}

\begin{tabular}{ll}
\hline ARTICLE INFO & ABSTRACT \\
\cline { 1 - 3 } $\begin{array}{l}\text { Received 07 April 2017 } \\
\text { Accepted 01 May 2017 }\end{array}$ & $\begin{array}{l}\text { Methods currently used in brain vascular disorder diagnostics are neither fast enough nor } \\
\text { clear-out; thus, there exists a necessity of finding new types of testing which could enlarge } \\
\text { ischemic stroke, } \\
\text { biomarker, } \\
\text { inflammation, } \\
\text { cytokines. }\end{array}$
\end{tabular} $\begin{aligned} & \text { discovery of sensitive and specific biomarkers of ischemic brain stroke will improve the } \\
& \text { effects of treatment and will help to assess the progress or complications of the disease. } \\
& \text { The relevant diagnosis of ischemic stroke (IS) within the first } 4.5 \text { hours after the initial } \\
& \text { symptoms allows for the initiation of treatment with recombinant tissue plasminogen } \\
& \text { activators which limits the magnitude of negative changes in the brain and which enhance } \\
& \text { the final effectiveness of therapy. The potential biomarkers which are under investigation } \\
& \text { are substances involved in the processes of coagulation and fibrinolysis, and are of } \\
& \text { molecules released from damaged vascular endothelial cells and from nerves and cardiac } \\
& \text { tissue. The analyzed substances are typical of oxidative stress, apoptosis, excitotoxicity } \\
& \text { and damage of the blood brain barrier. }\end{aligned}$

\section{INTRODUCTION}

By way of actual statistical data, diseases of the vascular system of the brain are the third most common cause of death in adults (after cancer and heart disease). Moreover, they are the major cause of permanent disability and lack of autonomy in adults. The incidence of stroke in Poland equals about 70 thousand cases per year.

According to the World Health Organization (WHO), a stroke is a syndrome characterized by the sudden appearance of a focal or generalized brain dysfunction, resulting from disorders of cerebral circulation which last more than 24 hours. The classification of strokes as based on patomechanism, divides strokes into ischemic, hemorrhagic and the very rare, subarachnoid hemorrhage. Approximately $85 \%$ of all cases are of ischemic stroke. This is caused by a blockage or a significant reduction in blood flow that lasts more than a few seconds. The main risk factors according to the TOAST classification (Trial of ORG 10172 in Acute Stroke Treatment) include changes in the large or small arteries supplying blood to the brain and congestions of a cardiac origin. Stroke risk factors can be either unmodifiable and modifiable, the latter being influenced by medical treatment and prophylactics. Unmodifiable risk factors include age, gender (male) and genetic factors; while the the modifiable are arterial hypertension, atrial fibrillation, hypercholesterolemia, diabetes, smoking status, excessive alcohol consumption, obesity and lack of physical activity.

Currently, the diagnosis of ischemic stroke is based on the clinical picture and neuroimaging studies of brain computed tomography (CT). The latter detects the presence or absence of intracranial hemorrhage. Basic laboratory tests, e.g. levels of glucose and electrolytes that reflects the general state of health, are also ordered because imbalance in these may cause focal neurological symptoms. If the ischemic stroke is diagnosed within 4.5 hours following the first symptoms, it is possible to give the patient a recombinant tissue plasminogen activator (rtPA). This dissolves the clot and restores cerebral perfusion. Of note, the accepted diagnostic procedure does not allow for the complete elimination of all diseases that can mimic stroke. Among these are associated migraine, transient ischemic attack (TIA), post-attack neuropathy, some brain tumors, certain metabolic disorders or multiple sclerosis $[15,17,18]$.

\footnotetext{
* Corresponding author

e-mail: krzysztof.szewczak@umlub.pl
} 


\section{PURPOSE OF THE SEARCH FOR NEW BIOMARKERS OF ISCHEMIC STROKE}

The necessity to look for new biomarkers of ischemic stroke (IS) comes from the need to rapidly facilitate and confirm an accurate diagnosis, to assess the complication's risk factors, to prevent death and to allow the introduction of an effective therapy. This is directly linked with the fastest possible response of medical personnel towards restoring the circulation in the oxygen-depleted area, and, thereby, reducing neurological deficits in patients.

Table 1. The division of biomarkers according to the direction of action

\begin{tabular}{|c|l|}
\hline No. & \multicolumn{1}{|c|}{ Types of the biomarkers } \\
\hline 1. & $\begin{array}{l}\text { Biomarkers useful for diagnosis of stroke - they help } \\
\text { to differentiate ischemic stroke from hemorrhagic } \\
\text { stroke and other diseases imitating stroke }\end{array}$ \\
\hline 2. & $\begin{array}{l}\text { Biomarkers helpful in the stroke's cause recognition } \\
\text { - they enable identification of the three main reasons } \\
\text { for stroke: changes in the large arteries supplying the } \\
\text { brain, changes in small arteries supplying the brain, } \\
\text { and cardiac congestion }\end{array}$ \\
\hline 3. & $\begin{array}{l}\text { Biomarkers helpful in the determination of the extent } \\
\text { of the impact (the degree of brain damage) - they } \\
\text { define the area of stroke and penumbra zone, and, } \\
\text { consequently, the degree of the patient's disability }\end{array}$ \\
\hline 4. & $\begin{array}{l}\text { Biomarkers helpful in prediction of risk for stroke } \\
\text { and relapse, as well - they help to enable the rapid } \\
\text { implementation of effective preventative treatment }\end{array}$ \\
\hline 5. & $\begin{array}{l}\text { Biomarkers useful to determine the direction of } \\
\text { treatment - they are useful during the qualification } \\
\text { of the patient for the use of an appropriate treatment }\end{array}$ \\
\hline
\end{tabular}

The National Institute of Health defined a biomarker as a biological characteristic that can be objectively determined. Such a kind of marker could be an indicator of physiological and pathological processes, but, as well, it may reflect the reaction to the therapeutic intervention. The ideal marker helpful in ischemic stroke recognition would be a single substance characterized by high specificity and sensitivity, that is detectable in blood or plasma by fast and noninvasive laboratory methods. In addition, the ideal indicator should predict the success of thrombolytic therapy, and also correlate with the size of the ischemia core, and, hence, provide the degree of disability and death risk. The biomarker types are listed in table 1. Past and current research has failed to identify an all-inclusive substance. The main barrier in finding the ideal biomarker is the heterogeneous structure of brain tissue, the cells of which respond in various ways to particular ischemia states. In addition, there are different causes of ischemia that influence disparity in the location, intensity and time of a stroke, and which are associated with varying degrees of severity of symptoms in patients. Another problem for fast recognition of biochemical substances in the blood stream is the brain-blood barrier. Biomarkers analyzed so far are indicators of subsequent phases of stroke; thus their applicability is limited. Taking into account all the above-mentioned difficulties in the current search paths, the aim of our work is to recognize the panel of biomarkers whose simultaneous determination would be useful in the early diagnosis of ischemic stroke (Fig. 1) [15,25].

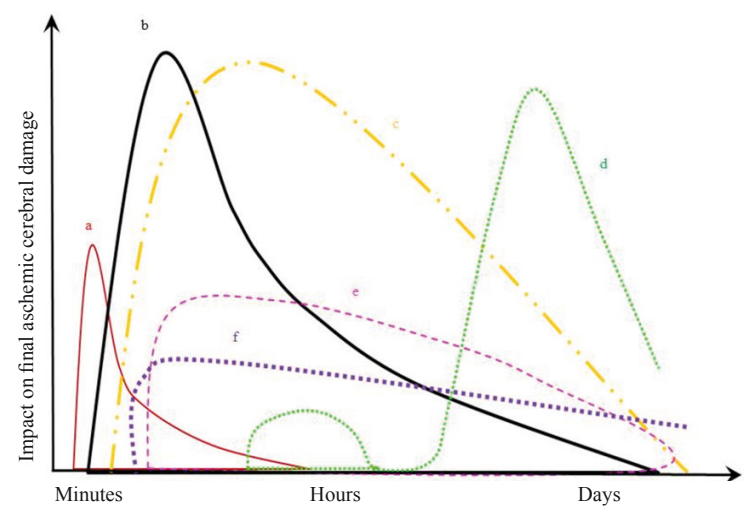

$\mathrm{a}$ - bioenergetic failure, $\mathrm{b}$ - excitotoxicity, $\mathrm{c}$ - oxidative stress, $\mathrm{d}$ - blood-brain barrier dysfunction, e - microvascular injury, $\mathrm{f}$ - hemostatic activation/postischemic inflammation [1]

Figure 1. Timing of events in the ischemic cascade

\section{POTENTIAL BIOMARKERS FOR ISCHEMIC STROKE}

The brain and neurons have little energy storage capacity, hence, a blockage of the blood supply carrying the oxygen and glucose causes infarction, and if the flow returns to normal before the death of a significant number of cells, the patient suffers only transient symptoms i.e. Transient Ischemic Attack (TIA). The target of the revascularization procedure is then to save the penumbra - the ischemic tissue surrounding the core of the infarct, the dysfunction of which could be reversible. The core of infarct is formed by diverse necrotic changes, due to the decrease of energy and due to apoptosis. The closing of the cerebral blood vessels is usually induced by thrombosis of the same vessels or by embolism from proximal segments of the heart arteries. In effect, the blood homeostasis is disturbed, and compensatory mechanisms such as fibrinolysis and coagulation, are activated (markers of fibrinolysis and coagulation processes). Afterwards, vessels injury induces an immune response manifested by the development of inflammatory response. The activated cells of the brain, as well as vascular cells, are involved in the synthesis of different molecules such as cytokines, chemokines, adhesion molecules and inflammatory enzymes - markers involved in the inflammation process.

The deprivation of glucose supply (energy) to neurons also leads to disturbances in the production of adenosine triphosphate - a marker of energy deficiency, which, in turn, results in malfunction of the ion pumps. The consequence of this is the depolarization of neurons, the increase of cellular sodium, calcium and chloride ions, as well as potassium ions in the extracellular space. Excess calcium leads to the activation of its dependent enzymes. These damage the cell membrane and the cytoskeleton, causing cell death. The activation of the excitotoxicity process succeeds though glutamate release from the synaptic ending, and by way of the activation of postsynaptic glutamate receptors. This causes the degradation of membrane lipids and the formation of free radicals that damage the brain cells. As a result of damaging factors such as metalloproteinase, the blood-brain barrier is injured and becomes permeable to leukocytes. This effect induces an inflammatory process at the infarct core area. 
The leaking blood-brain barrier, hence, enables the circulation between molecular mediators of the inflammatory cerebrospinal fluid (CSF) and the blood system $[13,15,17]$.

The contribution of inflammatory factors in ischemic stroke is considered through two aspects. The first is their impact on the occurrence of ischemia, especially with regarding to their participation in the development of atherosclerosis. This stands for $50 \%$ of all cases of ischemic strokes. The other is in their precipitation in the creation of the stroke core. During atherosclerosis, modified lipoprotein particles accumulate and form aggregates within the vessel intima. Lipoproteins possess a protein component called an 'apoprotein' (AI), which is responsible for lipids binding and metabolism. In the liver and the intestines, apoprotein AI (apoAI) is synthesized. This apoAI has a major role in the reverse cholesterol transport (RCT) from peripheral cells to the liver, while apolipoprotein B (apoB) is involved in cholesterol distribution to peripheral cells. Many independent studies have found that a high apoB/apoAI ratio is associated with an increased risk of ischemic stroke [4,14,29].

The accumulation of lipoproteins stimulates endothelial cells, macrophages and smooth muscle cells in the vascular wall to activate adhesion molecules: intercellular adhesion molecule 1 (ICAM-1), vascular cell adhesion molecule 1 (VCAM-1) and monocyte chemotactic protein (MCP-1). Those molecules, together with LDL damaged by free radicals (ox-LDL) and peripheral blood leukocytes, gather at the lesion site. According to actual data, such factors are risk factors for ischemic stroke [31].

The inflammatory process development in the wall of blood vessels is reflected by the elevation of proinflammatory cytokines: tumor necrosis factor- $\alpha$ (TNF- $\alpha)$, interleukin-6 (IL-6), interleukine-17 (IL-17), interferon- $\gamma$ (IFN- $\gamma$ ), as well as growth factors: insulin growth factor (IGF-1), platelet derived growth factor (PDGF), transforming growth factor (TGF- $\beta$ ) and vascular endothelial growth factor (VEGF). Those particles escalate the synthesis of C-reactive protein (CRP), fibrinogen, serum amyloid A (SAA), pentraxin-3 (PTX-3) and neutrophil gelatinase-associated lipocalin (NGAL). These 'acute phase proteins' aggravate the atherosclerosis process in the vascular bead. The level of TNF- $\alpha$ in serum and cerebrospinal fluid (CSF) correlates with the size of the ischemia and the stroke scale, according to NIHSS (Scale National Institutes of Health Stroke), while the acute phase of stroke is manifested by the elevated level of interleukin-1 receptor antagonist (IL-1RA) [27,37].

In addition to the CRP protein, Pentraxin - 3 (PTX-3) is an acute-phase protein. However, unlike CRP, it is not produced in the liver, but is generated locally in the site of an injury or an inflammation. PTX-3 synthesis is under the stimulating influence of interleukin $-1 \beta$ (IL-1 $\beta)$, TNF- $\alpha$ and the lipopolysaccharides, while interleukin - 4 (IL-4), interleukin - 13 (IL-13), and IFN- $\gamma$ inhibits PTX-3 production. The elevated level of pentraxin -3 is independently associated with increased mortality after ischemic stroke. Therefore, it could be used as a prognostic factor $[24,26]$.

Another known acute phase protein of interest is lipocalin neutrophil gelatinase-associated (NGAL). Its secretion increases during inflammation and endothelial damage. This protein is released from neutrophils and macrophages and hepatocytes, renal tubular cells, cells of the gastrointestinal and respiratory tract. NGAL binds to the metalloproteinase - 9 (MMP-9) and maintains its proteolytic activity by protecting it from degradation. It is also involved in apoptosis. Studies suggest that NGAL might be useful in distinguishing ischemic from hemorrhagic stroke [40].

The balance between inflammation and repair mechanisms determines the stable structure of atherosclerotic plaques. Nevertheless, it leads to slow and incremental narrowing of the vascular lumen. When the repair processes are weakened, the inflammatory mechanisms may be reinforced. This leads to the formation of unstable atherosclerotic lesions. A manifestation of this phase is the reduction of connective elements of the plaque and the development of its vascularity. Hence, inflammatory cells may penetrate into the core of the lesion. Macrophages and their proteolytic enzymes (the metalloproteinases), are responsible for the degradation of connective plaque elements. The enzymes release is controlled by TNF- $\alpha$, IL- $1 \beta$ and osteoprotegrin (OPG). Reports reveal that increased levels of MMP-9 and MMP-13 point to the plaque instability and to the risk for acute cerebrovascular episodes [2].

Osteoprotegrin is a soluble receptor classed within the superfamily of tumor necrosis factor receptors. OPG plasma concentration is also a marker of bone homeostasis, vascular calcification and the inflammatory state. Studies demonstrate a correlation between serum osteoprotegrin and the type of stroke. These also suggest a relationship with the risk of ischemia [12,30].

The acute inflammatory reaction is the response of an organism to the stroke, and the process of the acute phase has influence on the magnitude of the ischemia area. Cells that participate in such a reaction are classed as peripheral blood leukocytes. These flow to the damaged area via reperfusion and are generated by cells present at the site of the necrosis, e.g.: the neurons, astrocytes, microglia cells, endothelial cells, macrophages and lymphocytes T. The aforementioned cells are responsible for the production of inflammatory cytokines (TNF- $\alpha$, IL- 6 , IL-1) and are also a source of proteolytic enzymes, nitric oxide (NO) and free radicals, as well. The ischemia escalate and cover a larger and larger area, making the patient's state of health worse. These also initiate the activation and secretion of several chemotactic factors, such as the adhesion molecules responsible for leukocyte infiltration into the stroke's core. Moreover, these ischemia affect the production of the metalloproteinases, which induce the dysfunction of the blood-brain barrier and bring about increased endothelial permeability. The level of the prior-mentioned substances in the peripheral blood directly reflects the progression of inflammatory lesions during ischemia, and a direct relation between IL-6 level and the range of ischemia during the acute phase of stoke was reported $[5,35]$.

In the stroke area, aside from the proinflammatory cytokines, anti-inflammatory cytokines are also synthesized, among these, IL-10. During the acute phase of ischemia stroke, IL-10 is produced by peripheral blood monocytes, lymphocytes $\mathrm{T}$ and $\mathrm{B}$, macrophages and keratinocytes. The elevation of this cytokine was observed within the first 90 days from the beginning of ischemia. This situation suggests 
its involvement in neuroprotection and regeneration processes [6].

The balance between clotting and fibrinolysis is the basis of the vascular system homeostasis which provides defense mechanisms in the case of bleeding during trauma. Disturbances in hemostasis may have a strong impact on the occurrence of both stroke and myocardial infarction. Still, the clotting cascade can be induced even by a small vessel damage. This brings about further activation, as well as the adhesion and aggregation of platelets. The activation of endothelial cells and platelets during ischemic stroke is manifested by the elevation of several serum molecules. These include von Willebrand factor (vWF), selectin $\mathrm{P}$ and selectin E. The first, vWF, is an indicator of endothelial dysfunction, as it enables platelet adhesion to the endothelium. Moreover, vWF protects factor VIII against premature degradation. Studies show that the level of von Willebrand factor is increased in the acute and chronic stroke phase, and in patients with enhanced cardiovascular risk factors $[8,10]$.

Adhesion molecules include integrins, cadherins, immunoglobulin related molecules, ICAM, VCAM and selectins. Three types of selectins are distinguished: leukocytes - L-selectin (CD62L), endothelials - E-selectin (CD62E), and platelets $-\mathrm{P}$-selectin (CD62P). These participate in the process of leukocyte recruitment to the inflammatory area [42]. P-selectin is a protein accumulated in the $\alpha$-granules of platelets, and, together with vWF, in the endothelial cell Weibel-Palade body granules. This location facilitates a quick reaction of selectin $\mathrm{P}$ just after the stimulation of the clotting factors. Selectin then moves on the surface of endothelial cells, and, subsequently, to the cell membrane, where it mediates neutrophils and monocytes adhesion during an inflammatory reaction and clotting cascade. It is also involved in the formation of platelet-leukocytes aggregates, which leads to the release of prothrombotic tissue factors, cytokines and prothrombotic microplatelets. Studies show that the expression of P-selectin on the platelet surface is a marker of their activation. What is more, excessive activation of platelets in both the acute and chronic phase of stroke is manifested by an increase in the expression of $\mathrm{P}$-selectin (CD62P) and by elevated levels of circulating monocyteleukocyte complexes [19,41].

E-selectin mediates the adhesion of neutrophils to activated endothelial cells. It is mainly responsible for the transition from the "rolling leukocyte stage", to the closure of the adhesion stage of the same. Moreover, it is only under the impact of inflammatory mediators such as interleukins or TNF- $\alpha$ that endothelial cells are able to express E-selection. Most current research, however, puts forward that there is not any significant differences in the concentrations of E-selectin in patients with ischemic stroke and in controls (healthy individuals). Still, there are reports which suggest that selectin-E plays an important role in the pathogenesis of tissue damage after the ischemia and reperfusion stages. This indicates that the blockage of selectin-E would be useful in post-reperfusion treatment $[38,34]$.

Many studies confirm a significant increase of homocysteine concentration (Hcy) in patients with stroke [21,28]. Indeed, research holds that a high level of Hcy is an independent atherosclerosis risk factor and is associated with a twice higher mortality rate due to cardiovascular diseases. Due to its binding with NO, platelets aggregation increases and diastolic mechanisms of endothelia are impaired. Hcy may also have an influence on the clotting cascade, on tissue plasminogen activators and on apo-AI release, the final effect of which is the escalation of the clotting process and atherosclerosis.

A subsequent protein belonging to the coagulation cascade, and which may be a potential marker for ischemic stroke, is protein $\mathrm{Z}(\mathrm{PZ})$. It is a vitamin $\mathrm{K}$ dependent protein responsible for the degradation of the active $\mathrm{X}$ factor. It is suggested that a low level of this peptide may be associated with bleeding tendencies. However, clinical trials on humans after ischemic stroke have demonstrated conflicting results. On the one hand, the decreased level of protein $\mathrm{Z}$ may escalate the risk of stroke, while on the other hand, the elevation was found to be an independent risk factor for ischemic stroke. Further in-depth researches are necessary to clarify opinions about protein $\mathrm{Z}[9,33]$.

Cerebral infarction manifests by the activation of various processes in the brain tissue. Among these are neurodegeneration, demyelination and remyelination. Most researchers have confirmed the existence of a strong relationship between the release of substances from the damaged brain tissue and clinical status, as well as early and distant prognostics in ischemic stroke patients. Substances that are relatively well-known and noticed during ischemic stroke are markers of tissue damage and nerve cell and glial cell mortality. These include protein S100B, neuron specific enolase (NSE), myelin basic protein (MBP), protein tau, glial fibrillary acidic protein (GFAP), heavy and light neurofilaments (NF), and brain-derived neurotrophic factor (BDNF) - the last, a marker of the recovery mechanism $[11,23,36]$.

Because almost $30 \%$ of brain strokes are caused by heart dysfunction disturbances, potentially, heart functionality markers could be helpful in stroke recognition. Research data has shown the elevation of BNP (brain natriuretic peptide), NT-proBNP and HFAB after ischemic stroke. These results suggest that such proteins could be used as prognosis markers for earlier death in patients with transient ischemic attack (TIA) and stroke [7]. In the human organism, small intracellular proteins (FABP - fatty acid binding-proteins) bind long-chain fatty acids and then direct them to the appropriate places of intracellular recycling.

Heart fatty acid binding-proteins (HFAB), present in the cytoplasm of cardiac cells and the cells of kidney and brain, belong to the mentioned group. During ischemia, a rapid release of this protein occurs from damaged cells. This is released into the serum. However, some studies show contradictory reports concerning the HFABP marker and its usefulness in ischemic and hemorrhagic stroke differentiation $[22,39]$. In the cardiomyocytes of the heart chambers, under the influence of protease from the precursor pro-BNP, both BNP and the biologically inactive form, NT-proBNP, are produced. BNP is responsible for maintaining a balance of blood pressure and volume. Research has shown that during the acute stroke phase, patients had an elevation of BNP and NT proBNP as well. Moreover, a significant correlation of this indicator has been noted with ischemic area during stroke, as well as with the NIHSS scale [16,32]. 
Asymmetric dimethyl arginine (ADMA) is another protein correlated with the risk for cardiovascular disease. It is formed during the hydrolysis of proteins or polypeptides that contain methylated arginine, and is constantly produced in the course of the normal metabolism of proteins in many tissues, including vascular endothelial cells. Here, it inhibits nitric oxide synthase. An elevated level of ADMA may disturb NO synthesis, which leads to endothelial dysfunction, atherosclerosis and vascular disease. According to reports, elevated ADMA level might be particularly associated with a higher risk of cerebrovascular events [3,20].

\section{CONCLUSION}

Despite the clear definition of stroke and its generally accepted clinical treatment, approximately $25 \%$ of all diagnosed cases mimic the ischemic stroke. Many research centers are devoted to finding the ideal ischemic brain stroke biomarker, but, still, they have failed to reveal a sufficiently sensitive and specific marker or panel of it. Diagnostic difficulties arise from brain tissue heterogeneity, the existence of the blood-brain barrier and the complexity of processes during ischemia. It is reasonable, therefore, to conduct further research into the search for new substances that could be useful in the early diagnosis of stroke. This would limit the negative changes in the brain and contribute to the increased effectiveness of treatment.

\section{REFERENCE}

1. Brouns R., De Deyn P.P.: The complexity of neurobiological processes in acute ischemic stroke. Clin. Neurol. Neurosurg., 111, 483, 2009.

2. Castellanos M. et al.: Serum cellular fibronectin and matrix metalloproteinase- 9 as screening biomarkers for the prediction of parenchymal hematoma after thrombolytic therapy in acute ischemic stroke: a multicenter confirmatory study. Stroke, 38, 1855, 2007.

3. Chen S. et al.: Asymmetric Dimethyarginine as Marker and Mediator in Ischemic stroke. Int. J. Mol. Scien., 13, 15983, 2012.

4. Chorong H., Ki-Sook H.: Apolipoprotein B/A1 Ratio as Risk Factor for Cerebral Ischemic Stroke. Ewha Med. J., 35, 21, 2012.

5. Craig J.S. et al.: Peak plasma interleukin- 6 and other peripheral markers of inflammation in the first week of ischaemic stroke correlate with brain infarct volume, stroke severity and long-term outcome. BMC Neurology, 4, 2, 2004.

6. Fouda A.Y. et al.: Anti-inflammatory IL-10 is upregulated in both hemispheres after experimental ischemic stroke: Hypertension blunts the response. Exp. Transl Stroke Med., 5, 12, 2013

7. Greisenegger S. et al.: Biomarkers and Mortality After Transient Ischemic Attack and Minor Ischemic Stroke Population-Based Study, Stroke, 46, 659,2015

8. Hanson E. et al.: Plasma levels of von Willebrand factor in the etiologic subtypes of ischemic stroke. J. Thromb. Haemost., 9, 275, 2011.

9. Heeb M.J. et al.: Low protein $\mathrm{Z}$ levels and risk of ischemic stroke: differences by diabetic status and gender. Blood Cells, Mol. Dis., 29, $139,2002$.

10. Iskra T. et al.: Hemostatyczne markery uszkodzenia śródbłonka w udarze niedokrwiennym spowodowanym chorobami małych i dużych naczyń. Pol. Merkur. Lekarski, 21, 429, 2006.

11. Jauch E.C. et al.: Association of Serial Biochemical Markers With Acute Ischemic Stroke. Stroke, 37, 2508, 2006.

12. Jensen J.K. et al.: Osteoprotegerin concentrations and prognosis in acute ischaemic stroke. J. Intern. Med., 267, 410, 2010.

13. Jickling G.C., Frank R. Sharp F.R.: Biomarker Panels in Ischemic Stroke. Stroke, 46, 915, 2015.
14. Jong-Ho P. et al.: High Levels of Apolipoprotein B/AI Ratio Are Associated With Intracranial Atherosclerotic Stenosis. Stroke, 42, 3040, 2011.

15. Jóźwicka M., Głąbiński A.: The search for inflammatory biomarkers of ischaemic stroke. Aktualn. Neurol., 11, 106, 2011.

16. Kim M.H. et al.: Plasma biomarkers in the diagnosis of acut ischemic stroke. Ann. Clin. Lab. Sci., 40, 336, 2010.

17. Kim S.J., Moon G.J., Banga O.Y.: Biomarkers for Stroke. Journal of Stroke, 15(1), 27, 2013.

18. Lioutas V.A., Novak V.: Intranasal insulin neuroprotection in ischemic stroke. Neural. Regen. Res., 11, 400, 2016.

19. 19. Marquardt L. et al.: Course of platelet activation markers after ischemic stroke. Stroke, 33, 2570, 2002.

20. Nishiyama Y. et al.: Asymmetric dimethylaarginine (ADMA) as a possible risk marker for ischemic stroke. J. Neurol. Scien., 290, $12,2010$.

21. Palasik W.: Homocysteina - czynnik ryzyka występowania niedokrwiennego udaru mózgu. Postępy Nauk Medycznych, 14, 18, 2001.

22. Park S.Y. et al.: Plasma heart-type fatty acid binding protein level in acute ischemic stroke: comparative analysis with plasma S100B level for diagnosis of stroke and prediction of long-term clinical outcome. Cli. Neurol. Neurosurg., 115, 405, 2013.

23. Radwan W., Rabbah A., Saber H., Elghonemi M.: A new marker for ischemic cerebrovascular stroke: Phosphorylated Neurofilament $\mathrm{H}$. The Egyptian Journal of Critical Care Medicine, 1, 105, 2013.

24. Rodriguez-Grande B.: Pentraxin 3 mediates neurogenesis and angiogenesis after cerebral ischaemia, Journal of Neuroinflammation, 12, 15, 2015.

25. Rothstein L., Jickling G.C.: Ischemic stroke biomarkers in blood. Biomarkers in Medicine, 7, 37, 2013.

26. Ryu W.S. et al.: Pentraxin 3: a novel and independent prognostic marker in ischemic stroke. Atherosclerosis, 220, 581, 2012.

27. Shenhar-Tsarfaty S. et al.: Interleukin- 6 as an early predictor for one-year survival following an ischaemic stroke/transient ischaemic attack. Int. J. Stroke, 5, 16, 2010.

28. Shimizu H. et al.: Plasma homocysteine concentrations and the risk of subtypes of cerebral infarction. Cerebrovasc Dis., 13, 9, 2002.

29. Smolders B., Lemmens R., Thijs V.: Lipoprotein (a) and stroke: a meta-analysis of observational studies. Strok,. 38, 1959, 2007.

30. Song T.J. et al.: Association of plasma osteoprotegerin levels with stroke severity and functional outcome in acute ischaemic stroke patients. Biomarkers, 17, 738, 2012.

31. Tanne D. et al.: Soluble intercellular adhesionmolecule-1 and risk of future ischemic stroke: a nestedcase-control study from the Bezafibrate Infarction Prevention(BIP) study cohort. Stroke, 33, 2182, 2002.

32. Tomita H. et al.: Elevated plasma brain natriuretic peptide levels independent of heart disease in acut ischemic stroke: correlation with stroke severity. Hypertens. Res., 31, 1695, 2008.

33. Tran H.A., Eikelboom J.W.: Role of protein Z in stroke. Options Cardio. Med., 9, 191, 2007.

34. Tuttolomondo A. et al.: Plasma levels of inflammatory and thrombotic/fibrinolytic markers in acute ischemic strokes: relationship with TOAST subtype, outcome and infarct site. J. Neuroimmunol., 215, 84, 2009.

35. Waje-Andreassen U. et al.: IL-6: an early marker for outcome in acute ischemic stroke. Acta Neurol. Scand., 111, 360, 2005.

36. Węglewski A.: Biochemiczne markery uszkodzenia mózgu. Postępy Psychiatrii i Neurologii, 12, 111, 2003.

37. Welsh P. et al.: Associations of inflammatory and haemostatic biomarkers with poor outcome in acute ischaemic stroke. Cerebrovasc Dis., 27, 247, 2009.

38. Wiseman S. et al.: Blood Markers of Coagulation, Fibrinolysis, Endothelial Dysfunction and Inflammation in Lacunar Stroke versus Non-Lacunar Stroke and Non-Stroke: Systematic Review and MetaAnalysis. Cerebrovasc Dis., 37, 64, 2014.

39. Wunderlich M.T. et al.: Release of brain-type and heart-type fatty acid-binding proteins in serum after acute ischaemic stroke. J. Neurol., 252, 718, 2005. 


\section{Prospective of ischemic stroke biomarkers}

40. Yi-Chinn W., Wen-Hai Ch.: Neutrophil Gelatinase-Associated Lipocalin and Matrix Metalloproteinase-9 as Potential Biomarkers for Stroke: A Pilot Study. J. Neurol. Neurophysiol., 6, 2, 2015.

41. Yip H.K. et al.: Link between platelet activity and outcomes after an ischaemic stroke. Cerebrovasc. Dis., 20, 120-128, 2005.
42. Żerdziński M., Rychlik M., Partyka R.: Rola selektyn w rozwoju odpowiedzi zapalnej. Journal of Laboratory Diagnostics, 48, 347, 2012 